\title{
The intensity contrast of solar granulation: comparing Hinode SP results with MHD simulations
}

\author{
S. Danilovic ${ }^{1}$, A. Gandorfer ${ }^{1}$, A. Lagg ${ }^{1}$, M. Schüssler ${ }^{1}$, S. K. Solanki ${ }^{1}$, A. Vögler ${ }^{2}$, Y. Katsukawa ${ }^{3}$, and S. Tsuneta ${ }^{3}$ \\ 1 Max-Planck-Institut für Sonnensystemforschung, Max-Planck-Straße 2, 37191 Katlenburg-Lindau, Germany \\ e-mail: danilovic@mps.mpg.de \\ 2 Sterrekundig Instituut, Utrecht University, Postbus 80 000, 3508 TA Utrecht, The Netherlands \\ 3 National Astronomical Observatory of Japan, 2-21-1 Osawa, Mitaka, Tokyo 181-8588, Japan
}

Received 27 March 2008 / Accepted 16 April 2008

\section{ABSTRACT}

\begin{abstract}
Context. The contrast of granulation is an important quantity characterizing solar surface convection. Aims. We compare the intensity contrast at $630 \mathrm{~nm}$, observed using the Spectro-Polarimeter (SP) aboard the Hinode satellite, with the 3D radiative MHD simulations of Vögler \& Schüssler (2007, A\&A, 465, L43).

Methods. A synthetic image from the simulation is degraded using a theoretical point-spread function of the optical system, and by considering other important effects.

Results. The telescope aperture and the obscuration by the secondary mirror and its attachment spider, reduce the simulated contrast from $14.4 \%$ to $8.5 \%$. A slight effective defocus of the instrument brings the simulated contrast down to $7.5 \%$, close to the observed value of $7.0 \%$.

Conclusions. A proper consideration of the effects of the optical system and a slight defocus, lead to sufficient degradation of the synthetic image from the MHD simulation, such that the contrast reaches almost the observed value. The remaining small discrepancy can be ascribed to straylight and slight imperfections of the instrument, which are difficult to model. Hence, Hinode SP data are consistent with a granulation contrast which is predicted by 3D radiation MHD simulations.
\end{abstract}

Key words. Sun: granulation - Sun: photosphere

\section{Introduction}

The root-mean-square of the normalized continuum intensity fluctuations, within an area on the solar disk, is determined mainly by the intensity variation between the bright granules and the darker intergranular lanes, and thus is usually referred to as the granulation contrast. It is a key property of solar surface convection because it is connected with the temperature difference between rising (granules) and descending gas masses (intergranules), and thus related to the efficacy of the convective energy transport.

Reliable measurements of the granulation contrast are notoriously difficult, since the observed contrast suffers significantly from image degradation by the optical system and, most importantly in the case of ground-based telescopes, by seeing and straylight effects due to the terrestrial atmosphere. To deduce the "true granulation contrast" from measurements, we need to deconvolve the observed images by considering the modulation transfer through the optical setup (telescope, instruments, detector, etc.), in addition to through the terrestrial atmosphere. The quantitative effects of the latter are poorly understood and it is therefore unsurprising that the reconstructed values of the granulation contrast, documented in the literature, cover a broad range (see, e.g., Table 2 in Sánchez Cuberes et al. 2000). This remains true even if additional constraints, such as measurements of the intensity profile across the lunar limb during a partial eclipse, are used to derive the effective point-spread function (e.g., Levy 1971; Deubner \& Mattig 1975; Nordlund 1984; Sánchez Cuberes et al. 2000).
In the case of balloon-borne stratospheric observations, the influence of the atmosphere is negligible. Even under these conditions, however, the scatter of the reconstructed contrast values is large (Bahng \& Schwarzschild 1961; Pravdyuk et al. 1974; Altrock 1976; Edmonds \& Hinkle 1977; Wittmann \& Mehltretter 1977; Schmidt et al. 1979; Wittmann 1981), presumably because the proper consideration of the instrumental effects is nontrivial (Durrant et al. 1983).

Although the range of contrast values derived from observations is quite wide, the values predicted by $3 \mathrm{D}$ radiative HD/MHD simulations are significantly higher (e.g. Stein \& Nordlund 2000; Vögler et al. 2005; Wedemeyer-Böhm 2007). This is unsurprising, since the horizontal resolution of such simulations $(10-40 \mathrm{~km})$ is far better than what can be achieved observationally at present. To compare the observed contrast values with predictions from simulations, the synthetic images derived from simulations must be degraded in the same way as the observations, taking into account the effects of the optical system and, in the case of ground-based telescopes, the terrestrial atmosphere. This procedure is prone to the same uncertainties as the deconvolution of observations; the result depends strongly on assumptions about the nature of atmospheric seeing and straylight (Nordlund 1984; Schüssler et al. 2003; Rybák et al. 2006) and we are unable to infer if the simulation predictions are consistent with observations, or otherwise. However, the good agreement between observed and simulated spatially averaged spectral line bisectors indicates that the simulated intensity contrasts are probably close to actual values (Asplund et al. 2000). 
A new era has begun with the launch of the $50-\mathrm{cm}$ Solar Optical Telescope (SOT, Tsuneta et al. 2008) on the Hinode satellite (Kosugi et al. 2007). The good performance and low straylight level of the spectro-polarimeter (SP, Lites et al. 2001) and complete absence of atmospheric effects, enable a far more reliable determination of the granulation contrast at $630 \mathrm{~nm}$, the wavelength at which the SP operates. In this Letter, we compare the intensity contrast of a Hinode SP continuum map of the quiet Sun with predictions of MHD simulation.

\section{Observations and simulation data}

We determine the observed contrast from a map of the continuum intensity at $630 \mathrm{~nm}$ wavelength obtained on Jan. 16, 2007 (12:10:10-13:36:49 UT), using the scan mode of the Hinode SP with an exposure time of $4.8 \mathrm{~s}$ per slit position. The map covers a field of $163^{\prime \prime} \times 164$ " of "quiet" Sun, close to disk center. The slit width, the sampling step size, and the CCD pixel pitch all correspond to $0.16^{\prime \prime}$. The data were reduced using standard routines that correct for various instrumental effects (Lites et al. 2008). After reduction, we find an rms contrast of the continuum intensity of $7.0 \%$.

The 3D MHD simulation snapshot that we consider represents the saturated (statistically stationary) state of the dynamo run C of Vögler \& Schüssler (2007). The computational box contains $648 \times 648 \times 140$ cells; it corresponds to a physical size on the Sun of $4.86 \times 4.86 \mathrm{Mm}^{2}$ in the horizontal and $1.4 \mathrm{Mm}$ in the vertical direction, the latter ranging from about $900 \mathrm{~km}$ below to $500 \mathrm{~km}$ above continuum optical depth unity at $630 \mathrm{~nm}$ $\left(\tau_{630}=1\right)$. The simulation has been run with non-grey radiative transfer using the MURaM code (Vögler 2003; Vögler et al. 2005 ). With a horizontal grid-cell size of $7.5 \mathrm{~km}$, this simulation had one of the highest resolutions achieved so far. The snapshot that we use, has an average unsigned vertical magnetic field of about $7 \mathrm{G}$ at $\tau_{630}=0.1$, which we consider as a reasonable representation of the "quiet" Sun. The corresponding original (unsmeared) continuum image at $630 \mathrm{~nm}$ shows an rms contrast (standard deviation divided by mean value) of $14.4 \%$. The particular choice of snapshot was not critical for the results presented here. Considering a number of other snapshots and simulations of different spatial resolution or amount of magnetic flux in the simulation box, we found contrast values that were typically between $14 \%$ and $15 \%$.

\section{Modeling of the system PSF}

The difference between the observed and original contrast of the simulation amounts to more than a factor of two $(7.0 \% \mathrm{vs}$. $14.4 \%)$. Since there is no atmospheric distortion of the image, we investigate whether instrumental effects alone can account for this significant degradation.

The intrinsic resolution of the simulated images considered here (equivalent to $0.01^{\prime \prime}$ pixel size) is considerably better than the resolution of the 50-cm Hinode SOT; the degradation of the synthetic image based on the simulation result can therefore be modeled by applying an effective point-spread function (PSF). We convolved our simulated intensity maps in the 2D spatial domain with different PSFs, which represented a more and more realistic optical systems. To this end, we modeled the telescope and the transfer optical path down to the spectrograph entrance slit using the commercial optical design software $\mathrm{ZEMAX}^{1}$.

1 WWW . zemax . com

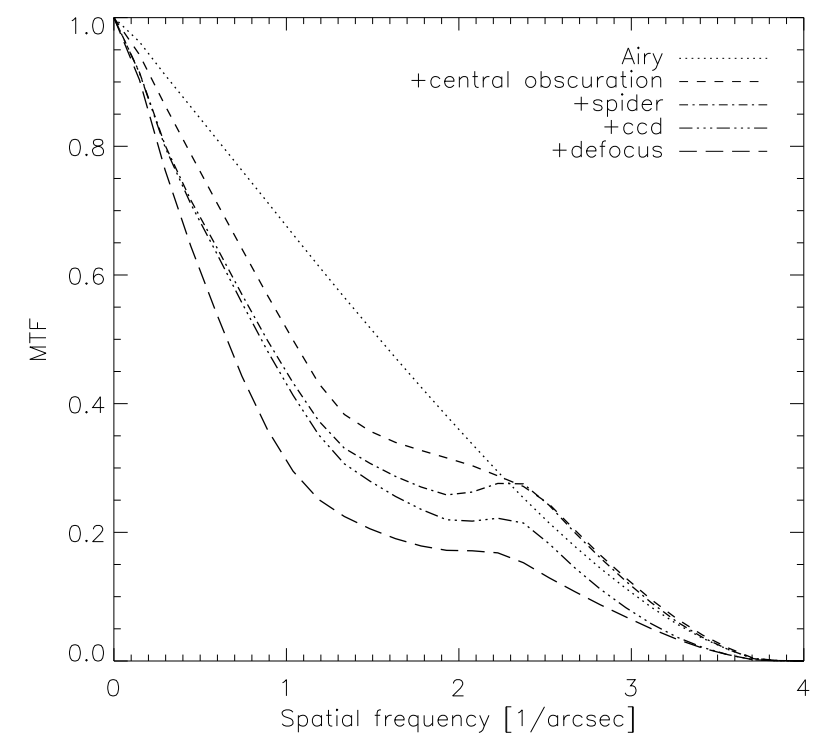

Fig. 1. Change of the Modulation Transfer Function when the effects of the different parts of the optical system are sequentially taken into account: telescope aperture ("Airy"), central obscuration, secondary mirror and spider, sampling (CCD), and a defocus of $1.5 \mathrm{~mm}$ (about 9 steps of the focus mechanism).

While the telescope was modeled with the nominal SOT surface parameters, the transfer (refocussing) path was modeled in paraxial approximation, which, however, has no influence on the final results of our calculations. ZEMAX calculates the PSF as the Fourier transform of the wavefront in the exit pupil of the system.

We describe the contrast degradation by the optical system using the Modulation Transfer Function (MTF), which is the Fourier transform of the point-spread function. In this way, the various effects can be considered step by step, as indicated below. Figure 1 shows the corresponding change of the system MTF.

Telecope aperture: the Hinode SOT is an $f / 9.1$ Gregory system with an aperture of $50 \mathrm{~cm}$, which corresponds to a cut-off at $1 / 0.26^{\prime \prime}$. Higher spatial wave numbers are not transmitted by the system, while low to intermediate wave numbers are transmitted with relatively high contrast.

Central obscuration and spider: important contributors to the contrast reduction are the central obstruction of the telescope by the secondary mirror, which produces a linear obscuration of $0.344=17.2 \mathrm{~cm} / 50 \mathrm{~cm}$, and the diffraction by the three spider elements, which are of $4 \mathrm{~cm}$ width each, holding the secondary. The effect of the central obstruction is that the highest spatial wave numbers are largely unaffected, while the intermediate wave numbers are significantly damped. The spider structure adds to this damping.

Spatial sampling: since the data considered here are obtained using the spectro-polarimeter, we have to consider the sampling and integration effects of the slit and the CCD detector. The sampling and the integration effects of the detector are approximated by powers of sinc functions (Boreman 2001).

Defocus: low-order optical aberrations damp the MTF at low spatial wave numbers. Since the Hinode Focal Plane Package (FPP) is a complex system, it is out of the scope of this Letter to model in detail all relevant contributions from optical, optomechanical, and electronic parts of the system. We therefore consider only the effect of a defocus in the plane of the SP slit. It turns out that a small amount of defocus can reduce significantly 


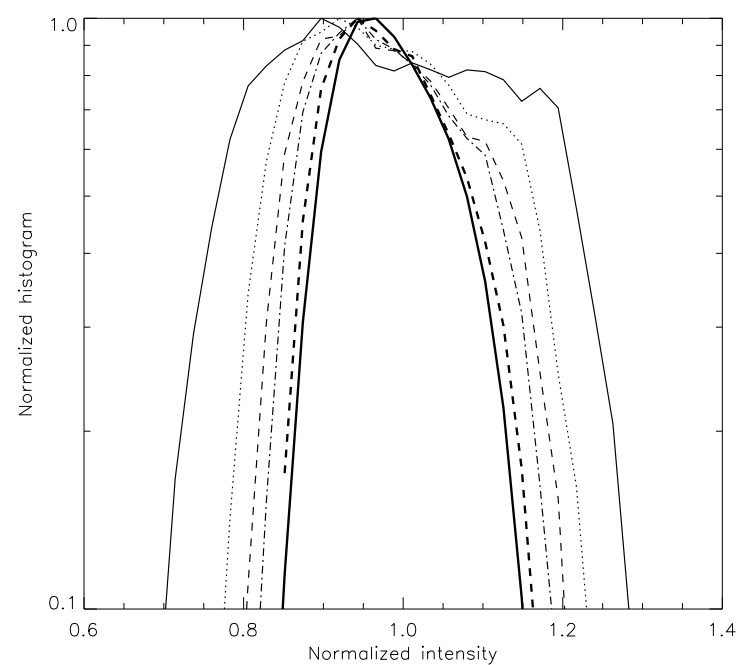

Fig. 2. Normalized histograms of the continuum-intensity values at $630 \mathrm{~nm}$, obtained for 35 equal bins between 0.6 and 1.4 of the mean intensity. Shown are results for the original synthetic image from the simulation snapshot (outermost, solid curve) and for the degraded images corresponding to the MTFs shown in Fig. 1 (same line styles as used there). The two thick inner curves represent the degraded synthetic image assuming a defocus of $1.5 \mathrm{~mm}$ (thick dashed curve) and the continuum map observed with Hinode SP (thick solid curve).

the intensity contrast, while leaving the fine-scale resolution of the system unaffected. The focus of the Hinode science instruments is controlled by means of a common reimaging lens that can be shifted in a range of $\pm 25 \mathrm{~mm}$, with a step of $0.17 \mathrm{~mm}$.

In addition, we investigated the effect of the (lossy) JPEG compression which was applied to the images. The change of the rms contrast turned out to be negligible, even for high compression factors.

\section{Degradation of the simulation data}

To compare with the Hinode result, we convolved the intensity maps calculated from the simulation with the different PSFs resulting from modeling the Hinode SOT as described in the previous section. After convolution, the degraded images were binned to represent the sampling and integration effects of the finite slit width, step size of the spatial sampling, and the pixel size.

Figure 2 shows the evolution of the histogram of intensity values in the simulation image as the various effects of the optical system are introduced. The rms contrast values for the various steps are listed in Table 1. The basic telescope effects (primary aperture, obscuration, and spider) reduce the rms contrast to $8.7 \%$, presumably since the small-scale intensity structure in the intergranular lanes is lost. There is almost no further contrast degradation due to sampling and rebinning (CCD), which indicates that the remaining contrast is determined mainly by spatial scales that are significantly larger than the critical sampling limit of the diffraction pattern. Once the fine detail in the granular lanes is no longer resolved, the contrast is dominated by the intensity differences between the granules and the average intensity of the intergranular lanes, which is almost unaffected by the integrating effect of the detector pixels. Similar values of the contrast reduction up to this step were obtained by Orozco Suárez et al. (2007).

The difference between the rms contrast degraded so far $(8.5 \%)$ and the observed contrast $(7.0 \%)$ is still significant. Another factor that contributes to contrast degradation is a slight
Table 1. Values of the rms intensity contrast after applying the PSFs corresponding to the MTFs shown in Fig. 1.

\begin{tabular}{lc}
\hline \hline Effects taken into account & rms [\%] \\
\hline none & 14.4 \\
Airy & 10.9 \\
+ central obscuration & 9.6 \\
+ spider & 8.7 \\
+ CCD & 8.5 \\
+ defocus $(1.5 \mathrm{~mm})$ & 7.5 \\
\hline
\end{tabular}

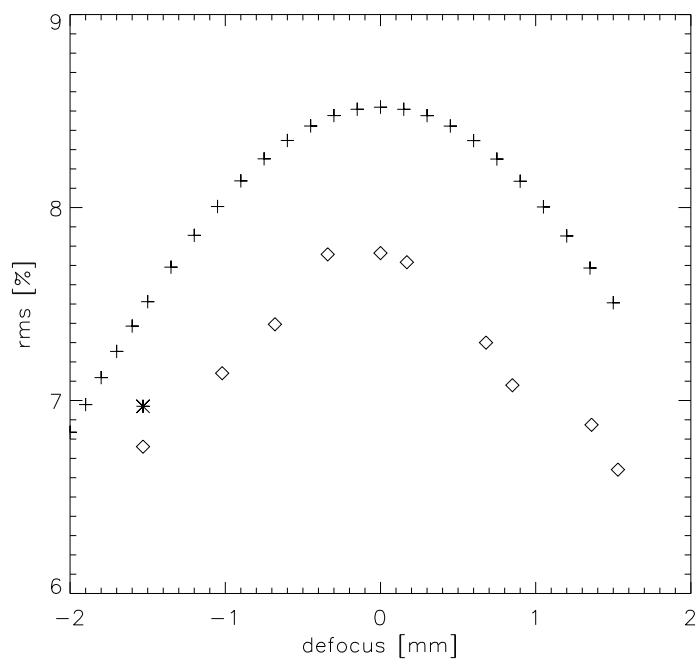

Fig. 3. Intensity contrast as a function of telescope defocus. Shown are results for a SP data set used taken on Jan. 16, 2007, 10:54:14-11:06:52 UT (diamonds) and the contrast values for the degraded simulation image (crosses). The value for the large map used to compare with the simulation is indicated by the asterisk, assuming that the location of the optimal focus was the same as for the other dataset. This also gives an indication of the observational uncertainty.

defocus of the SP, which affects mainly the low to intermediate spatial wave numbers. We have no direct information about the amount of defocus for the dataset considered here; we, however, obtained empirical evidence about the effect of defocus on the intensity contrast using a number of SP datasets for different focus positions, taken only an hour earlier than the map analyzed here. Apart from the exposure time of $1.6 \mathrm{~s}$ per slit position and the smaller size of the maps, all other observational parameters are the same. For every focus position, a map that covers a field of $3.2^{\prime \prime} \times 82^{\prime \prime}$ was generated. The corresponding contrast values given in Fig. 3 confirm the rather sensitive dependence on focus position. The figure also shows the dependence of the contrast of the simulation image on the defocus value. The large observational map with a contrast of $7.0 \%$ (indicated by the asterisk) was taken with a defocus of 8-9 steps $(1.36-1.53 \mathrm{~mm})$ from the optimal position in Fig. 3. The degraded simulation image corresponding to about this defocus value $(1.5 \mathrm{~mm})$ has a contrast of $7.5 \%$.

As an illustration of the image degradation, Fig. 4 shows the original continuum image from the simulation (left panel, the periodic simulation box is drawn fourfold for the sake of better visibility) and the final degraded image (middle). A subset of the observational map of the same size is shown in the right panel, using the same gray scale.

The remaining deviation of $0.5 \%$ from the observed value of about $7 \%$, and the general shift between the curves for simulation and observation in Fig. 3, can be ascribed to various 

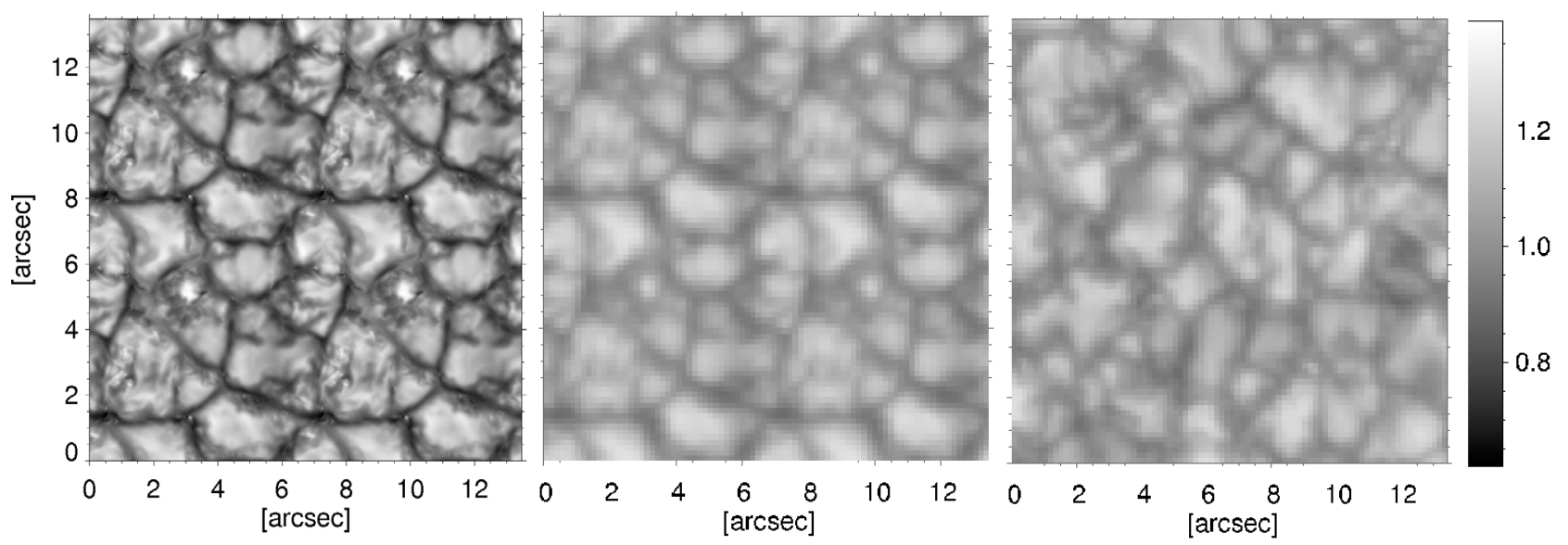

Fig. 4. Continuum images at $630 \mathrm{~nm}$ from the simulation snapshot with original resolution (left) and after degrading (middle), in comparison with a detail of the observed Hinode/SP map of the same size (right). The periodic simulation box is shown fourfold for better visibility.

degrading factors that are not included in our analysis. These include the effects of straylight, of all other low-order optical aberrations apart from defocus, and of the read-out electronics (such as transfer efficiency and pixel-to-pixel crosstalk), which reduce the contrast, but are difficult to model reliably. To illustrate the extreme sensitivity of the granulation contrast to loworder aberrations and straylight we calculated, as an example, in ZEMAX the effect of astigmatism and coma, which were equally distributed in such a way that the total system shows an rms wave-front error of 0.044 wavelengths. This corresponds to the number given by Suematsu et al. (2008) and represents a system with a remarkable Strehl ratio of 0.93. This small effect is nevertheless sufficient to reduce further the contrast from $8.5 \%$ to $8.1 \%$ in optimal focus position. If no further degrading effects are present, a straylight level of only $4.7 \%$ would be sufficient to bring the contrast down to the observed value of $7.8 \%$ (for optimal focus), and to $7.0 \%$ for the presumptive defocus value of the dataset studied here, respectively.

\section{Conclusion}

We find that consideration of the basic optical properties of the Hinode SOT/SP system and a slight defocus are sufficient to bring the degraded contrast of a 3D radiative MHD simulation and the observed rms continuum contrast at $630 \mathrm{~nm}$, almost into agreement. The remaining discrepancy can be ascribed to the combined, minor effects of straylight, other low-order optical aberrations, and instrument electronics. Hence, Hinode SP data are consistent with a granulation contrast at $630 \mathrm{~nm}$ of $14-15 \%$, as predicted by the simulations.

Acknowledgements. Hinode is a Japanese mission developed and launched by ISAS/JAXA, with NAOJ as domestic partner and NASA and STFC (UK) as international partners. It is operated by these agencies in co-operation with ESA and NSC (Norway).

\section{References}

Altrock, R. C. 1976, Sol. Phys., 47, 517

Asplund, M., Ludwig, H.-G., Nordlund, A., \& Stein, R. F. 2000, A\&A, 359, 669 Bahng, J., \& Schwarzschild, M. 1961, ApJ, 134, 337

Boreman, G. D. 2001, Modulation Transfer Function in Optical and ElectroOptical Systems (Bellingham), SPIE, TT52

Deubner, F. L., \& Mattig, W. 1975, A\&A, 45, 167

Durrant, C. J., Mattig, W., Nesis, A., \& Schmidt, W. 1983, A\&A, 123, 319

Edmonds, Jr., F. N., \& Hinkle, K. H. 1977, Sol. Phys., 51, 273

Kosugi, T., Matsuzaki, K., Sakao, T., et al. 2007, Sol. Phys., 243, 3

Levy, M. 1971, A\&A, 14, 15

Lites, B. W., Elmore, D. F., \& Streander, K. V. 2001, in Advanced Solar Polarimetry - Theory, Observation, and Instrumentation, ed. M. Sigwarth, ASP Conf. Ser., 236, 33

Lites, B. W., Ichimoto, K., Kubo, M., et al. 2008, Sol. Phys., submitted

Nordlund, A. 1984, in Small-Scale Dynamical Processes in Quiet Stellar Atmospheres, ed. S. L. Keil, Sunspot/NM: Sacramento Peak Observatory, 174

Orozco Suárez, D., Bellot Rubio, L. R., \& del Toro Iniesta, J. C. 2007, ApJ, 662, L31

Pravdyuk, L. M., Karpinskij, V. N., \& Andrejko, A. V. 1974, Solnechnye Dann. Bull. Akad. Nauk SSSR, No. 2, 70

Rybák, J., Kučera, A., Wöhl, H., Wedemeyer-Böhm, S., \& Steiner, O. 2006, in Solar MHD Theory and Observations: A High Spatial Resolution Perspective, ed. J. Leibacher, R. F. Stein, \& H. Uitenbroek (ASP), ASP Conf. Ser., 354, 77

Sánchez Cuberes, M., Bonet, J. A., Vázquez, M., \& Wittmann, A. D. 2000, ApJ, 538,940

Schmidt, W., Deubner, F.-L., Mattig, W., \& Mehltretter, J. P. 1979, A\&A, 75, 223

Schüssler, M., Shelyag, S., Berdyugina, S., Vögler, A., \& Solanki, S. K. 2003, ApJ, 597, L173

Stein, R. F., \& Nordlund, Å. 2000, Sol. Phys., 192, 91

Tsuneta, S., Suematsu, Y., Ichimoto, K., et al. 2008, Sol. Phys., in press [arXiv: 0711.1715v3]

Vögler, A. 2003, Ph.D. Thesis, Germany, University of Göttingen, http://webdoc.sub.gwdg.de/diss/2004/voegler

Vögler, A., \& Schüssler, M. 2007, A\&A, 465, L43

Vögler, A., Shelyag, S., Schüssler, M., et al. 2005, A\&A, 429, 335

Wedemeyer-Böhm, S. 2007, in Convection in Astrophysics, ed. F. Kupka, I.

Roxburgh, \& K. Chan (Cambridge Univ. Press), IAU Symp., 239, 52

Wittmann, A. 1981, A\&A, 99, 90

Wittmann, A., \& Mehltretter, J. P. 1977, A\&A, 61, 75 